\title{
Event Location and Vagueness
}

\author{
Andrea Borghini and Achille C. Varzi \\ Department of Philosophy, Columbia University, New York
}

[Final version published in Philosophical Studies 128:2 (2006), 313-336]

\section{Introduction}

That our event talk is vague is no news. Unlike facts, events are particulars located in space and time. But in ordinary circumstances it is utterly difficult, if not impossible, to specify the exact extent of the relevant spatiotemporal location. We say that Brutus stabbed Caesar and we intend to refer to an eventBrutus's stabbing of Caesar- that took place at a certain time in a certain place. It took place in the Senate (not in the Coliseum) during the Ides of March (not April) in Rome, 44 BC. But where exactly in the Senate did this event take place? Did it spread only through Brutus and Caesar? Did it spread through their entire bodies? (Was Brutus's left ear involved at all in this event?) Did it also spread through some space between them? Through what portion of space? And when exactly on March 15 did the killing begin? When exactly did it end?

We don't think the difficulty here is purely epistemic, as if it were just a matter of ignoring the facts. ${ }^{1}$ It's not that there is this event, Brutus's killing of Caesar, that has perfectly precise and yet unknown spatiotemporal boundaries, boundaries that historians have not been able (and will never be able) to locate. It's not that such events as the industrial revolution, the discovery of penicillin, or World War II have precise and unknown spatiotemporal boundaries whose location eschews us. The indeterminacy here is not epistemic, or so we claim. Does it follow that the indeterminacy is ontological - that events such as these have fuzzy spatial or temporal boundaries? We don't think so, either. Our difficulty in answering the above questions does not concern the ontological makeup of the relevant events. It concerns the structure of our event talk. It is the event designators we use that are vague, not the events themselves. ${ }^{2}$ It is conceivable that to each region of space-time there correspond one or more events of some sort. But our way of talking about such goings-on is very loose and, as a consequence, indeterminate.

Are events any different from objects in this regard? You point to an irregularly shaped protruding piece of land and say: 'Mount Everest'. Exactly what object are you referring to? What are its spatial boundaries? And what are its 
temporal boundaries - at least, what is the time of its coming into being? What are the spatiotemporal boundaries of the Mississippi River? Of downtown Manhattan? Of the cloud over the bell tower? Surely our object talk is also affected heavily by the phenomenon of vagueness. And surely one could give an account of such vagueness without blaming our ignorance and without implying that its source is ontological. There are plenty of physical objects out there-plenty of slightly distinct and yet precisely determinate chunks of matter. And when we say 'Mount Everest', or when we baptize a region 'Mount Everest', we are just being vague as to which of these objects we are referring to. Indeed, it is conceivable that to each region of space there corresponds, at any time, one or more objects of some sort, though our way of talking about such objects is very loose and, consequently, indeterminate.

There is, in fact, a striking similarity between the vagueness of our ordinary object talk and the vagueness of our event talk. And this similarity is reassuring if we think that events should be included in an inventory of the world. For, to the extent that we know how to handle the former - to the extent that the vagueness of our object-referring expressions does not seem to be a good reason to do away with the ontological category of objects ${ }^{3}$ - to that extent we can feel comfortable with the thought that the massive vagueness affecting our eventreferring expressions could be adequately handled without casting any doubts on the legitimacy of the ontological category of events. A supervaluational account, for example, could do the job in both cases: ${ }^{4}$ what we say, when we speak vaguely, is true if and only if it is true under every admissible semantic refinement (or "precisification") of our words.

Unfortunately, this is not the end of the story. In the case of events, it would appear that the vagueness bites deeper, and this is where one begins to worry. In the case of events the vagueness appears to bite deeper because it does not seem to stem exclusively from the vagueness of the words occurring in the expressions that we use to talk about them. This is especially clear if we consider that most - if not all - event-referring expressions are not proper names (whose referents could be arbitrarily refined) but definite descriptions obtained by nominalization of some event-reporting sentence. Characteristic of a description is that its referent depends on the meaning of its constituents, and it would be natural to expect that if the meaning of such constituents (names and predicates) were sharply defined, then so would be the referent of the whole description-hence its location. With objects this is the case, at least insofar as refining a predicate amounts to specifying a decision procedure for determining its extension. Thus, if the sortal 'cloud' were suitably refined, the description 'the cloud over the bell tower' would pick out a perfectly definite chunk of reality. If the name 'Manhat- 
tan' and the modifier 'downtown' were sharply defined, the term 'Downtown Manhattan' would pick out a definite region of space. And so on. Although in practice such refinements are out of reach, their availability as a matter of principle plays a significant role in our way of confining the vagueness of such expressions to the realm of semantics. By contrast, even if the semantics of every word we use were sharply defined, it would seem that an event-referring expression could still be vague in that it might still be indeterminate what region of space-time corresponds to the event in question. We may suppose that the exact reference of the two names, 'Brutus' and 'Caesar', is perfectly defined. And we may suppose that the extension of the relational predicate 'stabbed' is also perfectly precise, consisting of a certain set of well-defined ordered pairs among which, as it turns out, is the pair 〈Brutus, Caesar〉. We may thus, at least in principle, suppose that every term occurring in the sentence

\section{(1) Brutus stabbed Caesar}

is non-vague. Yet the event-referring description that comes from this sentence by ordinary nominalization would still be indeterminate: the spatiotemporal boundaries of Brutus's stabbing of Caesar would still be unsettled. And this appears to introduce a worrying asymmetry between ordinary event talk and object talk.

This line of reasoning is pressing. Nonetheless we think it can be resisted, and in the following we shall outline a way of resisting it. As long as we are clear about the context-sensitivity of our linguistic practices, we shall argue, the asymmetry turns out to be immaterial and a broadly semantic account of the relevant indeterminacy can be given in the case of events as well.

\section{Events and Participants}

There is, in fact, a simple way of restoring the symmetry that suggests itself immediately. Events have participants and these participants are spatiotemporally located. Hence, assuming this to hold generally, the question of an event's location could be settled indirectly by reference to the location of its participants. ${ }^{5}$ More precisely, one could rely on the following principle:

(A) The location of an event is the mereological sum of the locations of its participants.

This is especially plausible if we go along with the standard metaphysical picture, according to which an event is the exemplification of a property or relation 
by certain objects at a certain time. ${ }^{6}$ On this view, what an event is depends on what those objects - the participants - are. So it is reasonable to suppose that the location of an event, too, depends on that of its participants. Although this is not enough to justify (A), there is nothing implausible in this way of cashing out the relevant dependence vis-à-vis locative properties. If Brutus stabbed Caesar and nobody else took part in this event (as we may assume for the sake of simplicity), it is not implausible to suppose that the spatial location of the event referred to by the phrase 'Brutus's stabbing of Caesar' at any time $t$ during which this event took place is neither more nor less than the mereological sum of the spatial location of Brutus at $t$ and the spatial location of Caesar at $t .^{7}$ This still leaves room for indeterminacy with respect to the temporal location of the event, as the relevant period of time may still be vaguely defined. But that can be put aside. We may suppose that the predicate 'stabbed' is more informative in this regard than we have conjectured, so the answer might come from there. For any way of specifying a time of occurrence of an event, (A) would deliver a corresponding answer concerning its spatial location.

There is, unfortunately, an obvious difficulty that a solution along these lines must address. ${ }^{8}$ The difficulty arises insofar as the notion of an event participant may itself be up for grabs. Of course a full-blown metaphysical theory of events would say something about this - it would provide some means for answering the question of what it is for an object to qualify as an event participant. But even assuming that metaphysical question to be adequately answered, there remains the semantic question of how (or even whether) the participants of an event can be inferred from a linguistic report of the event itself. Suppose (1) is true: Brutus stabbed Caesar. Then it is plausible to suppose that Brutus was one of the entities involved in the stabbing - the agent, or subject - and Caesar was another one - the patient, or object. But if we are liberal enough with regard to mereological composition, then any mereological sum including Brutus would also qualify as a participant: it was involved in the stabbing after all, albeit only partially. Ditto for any mereological sum including Caesar. Indeed, if the universe as a whole were to count as an object, then it would take part in every event and (A) would imply that every event has the same (maximal) location. This would make (A) utterly useless.

An obvious suggestion would be to refine (A) in such a way as to rule out those participants that are not fully involved in the event. ${ }^{9}$ That is, we could distinguish between minimal participants, all parts of which are themselves involved in the event, and larger objects that only participate in the event by virtue of including a participant among their parts. Then we could refine (A) along the following lines: 
(A') The location of an event is the mereological sum of the locations of its minimal participants.

This proposal, however, would still be inadequate. Just as (A) suffered from the vagueness of 'participant', (A') obviously suffers from the vagueness of the minimality constraint. What exactly does it mean to be fully involved in an event? As already mentioned, perhaps not all of Brutus was relevantly engaged in the stabbing of Caesar (his left ear played no role, say). So it is not clear just how much of Brutus should be considered to individuate the location of the event. Even if we ruled out such worries by stipulation, assuming for instance that every stabbing must indeed be performed by a whole person, we could hardly count on stipulations being available in every case. Consider

(2) Titanic hit the iceberg and down it went.

There are two events involved here, a collision and a sinking. The sinking was of Titanic as a whole, that much is clear. It had exactly one participant, and it was Titanic. ${ }^{10}$ What about the collision-what were its participants? Well, every collision has at least two participants. And if we are talking about a collision between Titanic and the iceberg, then the natural thing to say is that the two participants are Titanic and the iceberg. Can we also say that they are the minimal participants of the event in question? Surely part of Titanic hit part of the iceberg. But there are huge parts of Titanic and huge parts of the iceberg that were not directly involved in the collision. They were affected by the collision, we may say, but they did not quite collide. And the fact that we synecdochically mention the whole of Titanic and the whole of the iceberg in order to pick out the collision should not confuse us any more than the fact that we may mention the collision in order to pick out those objects in their entirety. ('Titanic is that thing that collided against the iceberg'.) So neither Titanic nor the iceberg are good candidates for the minimal participants of the event in question. Are there any good candidates? Exactly what parts of Titanic hit what parts of the iceberg? Up to a point this is a matter of knowing the facts. But were the facts known-were we given a full report of that dramatic collision-we would still have difficulties answering the question. No non-arbitrary stipulations could do the job for us.

On the face of it, then, neither (A) nor ( $\left.\mathrm{A}^{\prime}\right)$ are adequate criteria for determining the location of every event. However, it may still be argued that either criterion represents a step forward vis-à-vis the cloud of suspicion that surrounds the event concept. For if something like (A) or (A') is assumed, then the vagueness involved in some of our event-referring expressions could be explained in terms of the indeterminacy affecting the notion of a (minimal) participant. This 
notion admits of several distinct precisifications, it could be argued, each of which is compatible with our linguistic practices. So a standard supervaluational account could be given after all. If every precisification yields the same location, then fine; otherwise the location is to be rated indeterminate because the competition among the admissible choices prevents us from settling the issue. (Never mind if you don't like supervaluational treatments of vagueness. Once the vagueness of 'participant' or 'minimal participant' is acknowledged, all sorts of semantic accounts can be implemented. We take supervaluationism to be the best option so we shall stick to it, but nothing significant will depend on this choice.)

\section{Modes of Precisification}

Let us, then, take a closer look at this suggestion. In order for it to be effective, we must be specific about the indeterminacy underlying the notion of a (minimal) participant. One way to cash out the nature of this indeterminacy is to say that the concept of a (minimal) participant is vague, so that (A) and (A') are at best vague criteria. This would place the burden of the indeterminacy on the theory of location that (A) and (A') aim to express. That is, the semantics of the object language could be treated along familiar lines, whether we are dealing with event- or object-referring expressions; yet a difference would show up insofar as the former, unlike the latter, turn out to be resistant to the advantages associated with the supposition that all vagueness can in principle be eliminated by suitably precisifying the vocabulary of the object language. If (A) and (A') are vague criteria, then our event talk can only be precisified in a vague manner.

This way of putting things is perfectly coherent, but we find it unsatisfactory as a matter of general methodology. A vague theory is not a good theory, and we should not buy into it unless we are forced to do so. We have said before that a full-blown metaphysical theory should provide some means for answering the question of what it is for an object to qualify as an event participant, hence we should better suppose that such means are precise or else we would be forced to admit some form of ontological vagueness. So here is a second, alternative way to cash out the nature of the indeterminacy underlying the notion of a (minimal) participant. The semantics of (A) and ( $\left.\mathrm{A}^{\prime}\right)$, we can say, is fine as it stands; it is the semantics of the object language that calls for revisions. With respect to (A), for instance, we need not say that the very notion of a participant is vague. We may hold instead that (A) is perfectly precise and insist that any extant vagueness lies in the terms and predicates that serve to pick out the relevant participants. (We will, in fact, stick to (A) from here on; the improvement represented by $\left(\mathrm{A}^{\prime}\right)$ is, from the perspective of the present proposal, negligible.) 
To illustrate, consider again a sentence such as (1). Our initial suggestion was that the exact location of the event reported by such a sentence ('Brutus stabbed Caesar') and denoted by the description that comes from it by nominalization ('Brutus's stabbing of Caesar') cannot be identified even if each term and predicate occurring in the sentence is assumed to be precise. However, we are now in a position to see that this suggestion was conceding too much. A precise definition of the extension of the predicate 'stabbed' must include the agent and the patient of each stabbing, and we could insist that such agents and patients should be neither more nor less than the relevant participants of the stabbings. Thus, if indeed the predicate 'stabbed' fully connotes the relation that is constitutive of the event in question, and if a precisification of this predicate includes in its extension the ordered pair 〈Brutus, Caesar〉, then it could be argued that such a precisification treats Brutus and Caesar as the relevant participants of the event in question. ${ }^{11}$ In that case, (A) would deliver a precise location for the event by identifying it with the mereological sum of the locations of Brutus and Caesar. But this is just one possibility. If the extension of the predicate were made precise by including, not the ordered pair $\langle$ Brutus, Caesar $\rangle$ but, rather, a slightly different ordered pair $\langle\alpha, \beta\rangle$ (with $\alpha$ and $\beta$ properly contained in Brutus and Caesar, respectively), ${ }^{12}$ then (A) would deliver a slightly different location for the event reported by (1). Each such precisification would yield a different answer to the question, Where did the stabbing take place? And if each precisification were equally acceptable, the corresponding multiplicity of answers would result in indeterminacy. Yet this indeterminacy would be perfectly standard, in that it would be explainable in terms of the indeterminacy of the object-language predicate 'stabbed'. Similarly, in the Titanic case, we could say that a multiplicity of precisifications is available for the predicate 'hit', some of which include in their extensions the ordered pair 〈Titanic, the iceberg $\rangle$ while others include in their extensions ordered pairs of the form $\langle\alpha, \beta\rangle$, with $\alpha$ and $\beta$ properly contained in Titanic and the iceberg, respectively. Because of this multiplicity, the location of the event would be indeterminate. But the indeterminacy would be purely semantic and would come entirely from the indeterminacy of the objectlanguage predicate 'hit'.

\section{Truth and Charity}

This alternative way of cashing out the vague location of an event strikes us as both intuitive and theoretically convenient. But there is still a complication. Suppose we say that Brutus and Caesar were only partly involved in the stabbing of the ides of March. (The story is perfectly parallel in the Titanic case, 
if one likes that example better.) That is, suppose we consider a precise interpretation of our language-i.e., a precisification $I$ of the interpretation $I_{0}$ fixed by our linguistic practices - according to which the extension of the predicate 'stabbed' includes, not the ordered pair 〈Brutus, Caesar), but a pair of slightly different objects, $\langle\alpha, \beta\rangle$. What are we to say of the sentence in (1)? If $I$ is indeed to be compatible with our linguistic practices, then this sentence should count as true under I: Brutus did stab Caesar. Yet a direct application of the standard truth-conditions for atomic sentences would, on the given precisification, yield a different answer. By the standard truth-conditions we mean the following:

(B) A sentence of the form ' $P\left(a_{1}, \ldots, a_{n}\right)$ ' is true, according to a (precise) interpretation $I$, if and only if the ordered $n$-tuple $\left\langle I\left(a_{1}\right), \ldots, I\left(a_{n}\right)\right\rangle$ is a member of $I(P)$.

And if 'Brutus' is interpreted as Brutus and 'Caesar' as Caesar, then (B) implies that (1) is false unless 'stabbed' is interpreted in such a way as to include the pair $\langle$ Brutus, Caesar - - which is not the case according to the precisification $I$ under consideration. So either we rule out $I$ as inadmissible - along with any other precise interpretation of the predicate 'stabbed' that fails to include the pair $\langle$ Brutus, Caesar〉, contrary to the suggestion that this predicate is relevantly vague - or something must be done about (B). Of course, we could also bite the bullet and concede that, strictly speaking, (1) is false under the given precisification $I$. Since there are also precisifications under which (1) is strictly speaking true (namely, those precisifications in which the extension of 'stabbed' does include the pair (Brutus, Caesar〉), this means that (1) would eventually be indeterminate. The supervaluation would fail to settle on a determinate truth-value. This is formally impeccable. But as an account of the matter it is utterly unsatisfactory. If we are serious about ordinary language - if we want to preserve the data insofar as these are fixed by our linguistic practices, at least when these practices are not overtly inconsistent - the truth of (1) is non-negotiable. Ditto for (2).

Thus something must be done about (B). This complication is serious because (B) is a fundamental principle of extensional semantics. However, independently of the worry at issue, it should come as no surprise that (B), as any principle of formal semantics, involves simplifications that in actual circumstances may not be fully fulfilled. Here the underlying presupposition is that an atomic sentence of the form ' $P\left(a_{1}, \ldots, a_{n}\right)$ ' provides a complete report of a state of affairs. Yet in ordinary circumstances this may not be the case, either because the predicate ' $P$ ' gives only an approximate connotation of the relation linking 
the objects referred to by the singular terms ' $a_{1}$ ', $\ldots$, ' $a_{n}$ ', or because the terms themselves pick out the relevant objects with a certain degree of approximation. In assessing the truth of a sentence such as

(4) John's glass is empty,

for example, we are supposed to construe the predicate 'empty' charitably, allowing for the possibility that there is a straw inside the glass, or that the glass is filled with air, or that it still contains a few drops of water. If we just looked at the extension of 'empty', strictly construed, we might not find John's glass among its members, but this is no reason to regard (4) as false. Similarly, and more to the point, in a sentence such as

\section{(5) John's bicycle is red,}

the natural thing to do is to construe the referent of 'John's bicycle' charitably. Presumably not all of the bicycle is red, but only the frame, possibly only the outer surface of the frame. In ordinary circumstances we need not be too specific about such matters, so we don't bother. We often speak synecdochically. But a straight application of (B) would deliver the wrong results unless we take such matters into account.

Now, one conservative solution here would be to say that the issue is to be dealt with at the level of logical form, without affecting the standard formulation of the semantic principle in (B). For instance, we could insist that, once the context is fully clarified, an utterance of (5) should be interpreted as elliptic for something like

(5') The outer surface of the frame of John's bicycle is red.

And the truth-conditions for this sentence could be handled directly by (B): (5') would be true if the extension of 'red' includes the outer surface of the frame of John's bicycle, and false otherwise. If this account is accepted, then we may insist that the case of such sentences as (1) and (2) is no different. We might insist that, on closer inspection, an utterance of such sentences should be interpreted as elliptic for something else. In these cases the context might not be informative enough to tell us exactly what part of Brutus stabbed what part of Caesar or what part of Titanic hit the iceberg. But we might still consider reinterpreting our sentences as elliptic existential generalizations:

(1') A certain part of Brutus stabbed a certain part of Caesar.

(2') A certain part of Titanic hit a certain part of the iceberg, and down went Titanic. 
This would be enough. For we are thinking of evaluating our sentences with regard to precisifications that interpret 'stabbed' and 'hit' as relations that hold true of certain ordered pairs of objects, $\alpha$ and $\beta$, properly included in Brutus and Caesar, or in Titanic and the iceberg, respectively. And the semantics for the existential quantifiers in (1') and (2'), together with (B), would yield the expected values.

If this conservative account of the matter is accepted, then the complication dissolves and we are done. The sort of charity required to do justice to our intuitive assessment of the truth-values of such sentences as (1) and (2) would be taken care of at the level of logical form, prior to the application of our formal semantic tools, and this would in no way conflict with the (semantic) account of the indeterminacy affecting the location of the events reported by such sentences. The location would be indeterminate precisely because the hidden existential quantifiers fail to single out the event participants in a determinate way. On the other hand, this conservative account faces some problems of its own. For one thing, the appeal to logical form may be deemed unattractive on various grounds. (Can it be generalized to all cases?) More importantly, its material adequacy is doubtful: whatever parts of Brutus and Caesar qualified as truth-makers for (1), interpreted as (1'), would also qualify as truth-makers for a host of other sentences, interpreted accordingly. And this would take us back to the general problem raised by (A). For example, assuming that Caesar is part of the population of Rome, the truth of (1), interpreted as (1'), would imply the truth of

(6) Brutus stabbed the population of Rome,

interpreted as

(6') A certain part of Brutus stabbed a certain part of the population of Rome.

(The implication would follow from the transitivity of 'part of'.) But this is idle: sentence (6) is plainly false no matter how one understands it. ${ }^{13}$

So here is a less conservative option. Let us openly acknowledge the limits of (B) and let us account for the charity required by our linguistic practices directly at the semantic level. In the terminology used above, this means taking seriously the suggestion that in ordinary circumstances we help ourselves with singular terms that pick out the referents of our discourse with some degree of approximation - not because such terms are vague but because the intended referents are vaguely understood. We say 'John's bicycle' meaning some relevant part of the bicycle; we say 'Brutus' meaning some relevant part of Brutus; we say 'Titanic' meaning some relevant part of the ship; and so on. The sort of 
relevance at issue is not specified and in this sense the intended referents are only vaguely understood. In ordinary circumstances precision hardly matters, hence we generally do not worry about this kind of vagueness. But we do need to worry about it when it comes to the task of formally applying a principle such as (B).

To spell out this suggestion more formally, let $I$ be a precise interpretation of the language, possibly a precisification of a given vague interpretation. It assigns a referent $I(a)$ to every singular term ' $a$ ' and an extension $I(P)$ to every predicate ' $P$ '. A charitable reading of $I$ may be construed as an interpretation $I^{*}$ that yields distinct but suitably related values: each $I^{*}(a)$ may be a slightly distinct object from $I(a)$ (for instance, a certain proper part of $I(a)$ ) and each $I^{*}(P)$ might be a set of slightly distinct tuples from those contained in $I(P)$. Then the natural thing to do is to revise (B) as follows:

(B') A sentence of the form ' $P\left(a_{1}, \ldots, a_{n}\right)$ ' is true, according to a (precise) interpretation $I$, if and only if there is a charitable reading $I^{*}$ for $I$ such that the ordered n-tuple $\left\langle I^{*}\left(a_{1}\right), \ldots, I^{*}\left(a_{n}\right)\right\rangle$ is a member of $I^{*}(P)$.

(The charitable reading of the singular terms is meant to take care of such cases as (1), (2), and (5); the charitable reading of the predicates is to take care of cases such as (4).)

Of course, the notion of a charitable reading is heavily context-dependent and vague. But this is no big deal. The notion of an admissible precisification involved in the basic supervaluational rule,

(C) A sentence is true, according to an interpretation $I_{0}$, if and only if it is true according to every admissible precisification $I$ of $I_{0}$,

is equally vague. This is where the phenomenon of higher-order vagueness kicks in. Likewise for $\left(\mathrm{B}^{\prime}\right)$. Higher-order semantic indeterminacy is hardly a peculiarity of our event talk, so from the present perspective we should not worry about it. (By contrast, the indeterminacy involved in the minimalist criterion ( $\left.\mathrm{A}^{\prime}\right)$, if understood as a vague criterion as indicated at the beginning of section 3 , would be peculiar to our way of understanding event talk and for that reason should be avoided.) Note, however, that the notion of a charitable reading is significantly different from the notion of a precisification. The latter picks out, compatibly with our linguistic practices, a precise referent and a precise extension for each vague name and predicate of the language. The former picks out, for each such precise interpretation, a contextually plausible variant. Thus, the name 'Brutus' might be vague in that it may not be clear whether certain bits and pieces are to be included in its referent. (Is the cherry that Brutus is presently chewing part of 
him? Will it be part of Brutus only after he has swallowed it? After he has digested it?) Any admissible way to resolve this vagueness corresponds to a precisification of 'Brutus'. And for each such precisification $I$ there may be various charitable readings whereby the referent of 'Brutus' is interpreted in yet another, slightly different way, depending on the context of use of the name.

So this is how we think the complication can be handled semantically. To determine the participants of an event we need consider different ways of precisifying the predicate connoting the property or relation whose exemplification the event is. Each such precisification, in turn, requires that the singular terms purporting to denote the participants of the event be understood charitably, and (B') provides us with the relevant revision of the standard, uncharitable semantic rule (B). How exactly charitable readings should be construed is of course a difficult question and much work would at this point be required in order to provide a thorough account. This may well be a task to be carried out in a case-by-case fashion. But we need not spell out the details here. For if the analysis of such sentences as (4) and (5) is correct, then some charity appears to be needed across the board anyway. It is not just a complication in the semantics of our event talk but it affects the semantics of our object talk as well. And that is fair enough: our purpose is precisely to show that both sorts of talk are, from a semantic perspective, on equal footing.

\section{Two Final Worries}

To sum up, then, our proposal involves two main steps. First, we rely on (A) (or $\left(A^{\prime}\right)$ ) to identify the location of an event with the mereological sum of the locations of its (minimal) participants. Next, we identify the participants of an event with the entities that make up the extension of the predicate (e.g., 'stabbed') used to pick out the event-constitutive property or relation. If this predicate is vague, its extension may not be uniquely defined and, consequently, the participants may not be uniquely identified either, which will induce a corresponding indeterminacy in the location of the event. But this indeterminacy is thoroughly semantic, precisely insofar as it depends on the semantic indeterminacy of the relevant predicate. In addition, we also propose (third) to supplement this account with a modification of the standard truth conditions for atomic sentences of the form ' $P\left(a_{1}, \ldots, a_{n}\right)$ ', among which those sentences that report the occurrence of an event. Not only should such sentences be evaluated with respect to the admissible precisifications of the given interpretation $I_{0}$ of the language - for instance according to the supervaluational rule (C); relative to each precisification $I$ of $I_{0}$, they should be evaluated with respect to a charitable reading $I^{*}$ 
of $I$-as per rule $\left(\mathrm{B}^{\prime}\right)$. This is not necessary to explain the vagueness associated with the location of an event, but it is necessary to ensure that the explanation itself does not conflict with the semantic intuitions underlying the corresponding event-reporting sentence.

Is this account general enough? Can every event-referring expression be handled along these lines? As we see it, the account works well for those eventreferring expressions that come from nominalization of an event-reporting sentence, as 'Brutus's stabbing of Caesar' comes from nominalization of 'Brutus stabbed Caesar', on the assumption that the predicate used in the sentence (here: 'stabbed') fully connotes the property or relation that is constitutive of the event in question. But there are two residual worries. First, it is a fact that many ordinary event-referring expressions are not of this sort. Consider again 'the industrial revolution' or 'World War II'. What are the object names and predicates whose semantics can deliver the (vague) answer to the location question for the events picked out by these expressions? Second, even when dealing with eventreferring expressions that do come from nominalization, there is no guarantee that the relevant predicate always fully connotes the event-constitutive property or relation. In fact we have better not make that assumption. Otherwise one might be inclined to conclude that insofar as

(1) Brutus stabbed Caesar.

involves a two-place predicate while

(7) Brutus stabbed Caesar with a knife.

involves a three-place predicate, the events picked out by the corresponding nominalizations would involve a different number of participants and would therefore be distinct - contrary to intuition. Likewise, we would have to distinguish between such events and the events reported by

(8) Brutus lowered his right arm.

(9) Brutus committed homicide.

(10) Brutus set a bad example for others to follow.

and so on - again contrary to intuition. At least, this would run against the intuition - which we share - that one and the same event can be re-described in different, non-equivalent ways. ${ }^{14}$

So more work is needed. Here we shall confine ourselves to some brief remarks, with no pretense of completeness. Concerning the first worry-that not every event-referring expression comes from nominalization-it must be acknowledged that our account has no straightforward answer to offer. No object 
names and predicates are available whose semantics can account for the (vague) location of the events picked out by such descriptions as 'the industrial revolution' or 'World War II'. However, let us point out that such descriptions are typically intended to pick out complex events, i.e., events consisting of a multiplicity of simpler ones, for which a canonical description by nominalization is in principle available. What these simple events are is only vaguely understood, and it is in this sense that the descriptions of the relevant complex events are vague: there are many collections of little episodes that could legitimately qualify as the constituents of the industrial revolution - many distinct mereological aggregates of events that could legitimately qualify as the referent of the term 'the industrial revolution' - and when we use this term we are just being vague as to which of those collections or aggregates we are referring to. Our linguistic practices do not single out a unique referent. But this is no further problem. It is, in fact, the exact analogue of the sort of vagueness that we run into when we use such vague object designators as 'Everest' or 'Downtown Manhattan'. And for such vagueness a straightforward supervaluational account is already available.

Thus the first worry boils down to this: Are there any event descriptions that do not come from nominalization and yet are not meant to pick out complex events? It's hard to deny this as a matter of principle. The question of whether there are any simple, atomic events is by itself a difficult metaphysical question and it would be presumptuous to build the answer into a semantic account of ordinary language. But we conjecture that the simpler an event is - the closer it is to being a simple property-exemplification - the more likely it is that among our means for referring to it are descriptions that come from nominalization. Modulo this conjecture, we are therefore inclined to think that the worry can be met.

One might still object that the conjecture itself, even if accepted, is of dubious relevance. ${ }^{15}$ Consider a description that refers to an event indirectly-e.g., by temporal relation to another event, as in 'what Brutus did after Cassius handed him the knife'. If such a description is meant to pick out a simple event, we are suggesting that an alternative, canonical description of that event can be obtained by nominalization (for instance, the description 'Brutus's stabbing of Caesar') for which our semantic account is available. Yet it might be observed that whether such an alternative description is indeed adequate depends on whether it is co-referential with the original, non-canonical description. And how can that be established if we are talking about descriptions that are vague? More importantly, if the original description is vague, how can we give a semantic account of its vagueness by appeal to the vagueness of terms and/or predicates that do not even appear in that description? 
Our reply is that nothing peculiar here seems to hinge on the fact that we are talking about descriptions of events. We may ask the same questions with respect to object descriptions (or even names), and in such cases the answers seem easily available. The terms 'Mount Everest' and 'the tallest mountain' are both vague, yet we are perfectly justified in assuming that they are co-referential. More importantly, when it comes to their semantics we are justified in explaining the vagueness of one term directly, and then in relying on that explanation to account for the vagueness of the other precisely in virtue of their co-referentiality. If so, then there is in principle nothing illegitimate in supposing that two vague event descriptions may be co-referential, or in explaining the vagueness of one via the vagueness of the other. It may be hard to find a good canonical description for an event that is vaguely described in a non-canonical way. But we often find it, and when we do we know how to handle the relevant vagueness. ("What Brutus did after Cassius handed him the knife was atrocious." "Why, what did he do?" "He stabbed Caesar" "And where did that happen?" Well, it depends on how exactly you understand the predicate 'stabbed'...")

Moving now to the second worry - that even a canonical event-referring expression obtained by nominalization may involve a predicate that fails to fully connote the event-constitutive property or relation - several cases may be distinguished. Consider first the case illustrated by the opposition between (1) ('Brutus stabbed Caesar') and (7) ('Brutus stabbed Caesar with a knife'). One plausible view is that only one of these two sentences involves a fully connoting predicate. One may take this to be the binary ' $x$ stabbed $y$ ' (as we have been doing in the foregoing) and construe (7) as a sentence in which the stabbing is said to have occurred and to be correlated to the knife by the instrumental tie expressed by 'with'. In this case it is certainly possible to understand both sentences as describing one single event with two participants, and the logical nexus between (1) and (7) would be one of standard adverbial modification. ${ }^{16}$ Conversely, one may take the fully connoting predicate to be the three-place ' $x$ stabbed $y$ with $z$ ' and construe (1) as elliptic for

(1") Brutus stabbed Caesar with something,

in which case both (1) and (7) would be about an event with three participants and the relevant logical nexus would be one of existential generalization. ${ }^{17}$ Both options are plausible, and both are compatible with the account offered here.

Now consider the opposition between (1) and, say, (8) ('Brutus lowered his right arm'). If we think these sentences report different events, then fine. But if we want to regard both sentences as reporting the same event, then we face a greater difficulty because the predicates they involve are logically independent 
of each other. We may still insist that one of these sentences tells the whole truth about what happened (albeit vaguely) and provide a suitable paraphrase of the other. (For instance, if we think that 'stabbed' fully connotes the relevant eventconstitutive relation, we may say that (8) is elliptical for something like

(8' ) Brutus lowered his right arm upon Caesar,

where 'lowered his arm upon' is a predicate that only partially connotes the relation connoted by 'stabbed'.) But in general there is no reason to think that such a paraphrase is always available, let alone plausible. Rather, it seems to us that such cases suggest a different diagnosis. We just have to acknowledge that when a predicate does not fully connote, or does not connote at all, the relevant eventconstitutive property or relation, its semantics may not be informative about the identity (and location) of the reported event. We can still form a description of the event by ordinary nominalization. But this description may be as misleading - semantically - as any description that refers to the event indirectly, as with 'the industrial revolution' or 'what Brutus did after Cassius handed him the knife'. To put it differently, our account has been developed with respect to canonical event-descriptions obtained by nominalization of atomic sentences in which the predicate is assumed to fully connote the property or relation that is constitutive of the reported event. When this assumption is not met, the nominalization is semantically uninformative. It may be that in spite of the appearances we are dealing with a complex event, in which case we have already seen how to proceed. (Consider 'the discovery of penicillin'; although grammatically this description comes from nominalization of 'Penicillin was discovered', it can hardly be regarded as referring to a simple event and its semantics is best treated in analogy to 'the industrial revolution'.) Or perhaps we are dealing with a simple event, but an event whose canonical description is quite different, in which case we have to come up with that description before providing a detailed semantic account along the lines suggested here. Indeed, we are inclined to think that we often find ourselves in this situation. The metaphysical thesis that an event is the exemplification of a certain property by certain objects at a certain time does not imply that every expression referring to that event involves names and predicates that aim to pick out the relevant property and objects, even when the expression is - grammatically - the result of a nominalization. When it does not involve such names and predicates, our semantic account can only be applied indirectly.

These limitations are not to be underestimated. They show that the semantics of our event talk is indeed quite a complex matter. Nonetheless we conclude that in view of the account offered above the asymmetry between objects and 
events vis-à-vis the phenomenon of vagueness loses its force. There certainly is a greater difficulty in determining the reference of our event talk. But the difference is one of degree and should not be given theoretical weight. What event an expression is about is not something that can be accurately inferred from the words we use; it depends heavily on context and unprincipled intuitions - much more heavily than with ordinary object talk. This results in massive semantic vagueness. But this is no reason to do away with events altogether, just as the vagueness of our object talk is no good reason to do away with the ontological category of objects. $^{18}$

\section{Notes}

${ }^{1}$ The epistemic account of vagueness, in general and presumably also in cases such as these, is defended e.g. by Sorensen (1988) and Williamson (1994).

${ }^{2}$ Here and below we speak of 'event designators' in the broadest sense, to include any expressions that can be used to refer to individual events. We doubt there are any logically proper names of events, though. In those few cases where grammatical proper names are used - e.g., to refer to hurricanes - they arguably function as disguised definite descriptions. (See Bennett 1988: Ch.1).

${ }^{3}$ With some exceptions-e.g., Unger (1979). Indeed, Unger's view is not as idiosyncratic as it might sound. Several defenses of the four-dimensionalist conception of objects (e.g., Heller 1990 and Sider 2001) arise from similar concerns.

${ }^{4}$ On this we refer to Varzi $(2001,2002)$, respectively. The supervaluational account of vagueness goes back to Fine (1975); its application to singular terms stems from Lewis (1988).

${ }^{5}$ Some philosophers have questioned the assumption, citing as counterexamples changes in light or weather conditions (Brand 1977: 335) or, perhaps more plausibly, flashes and bangs (Strawson 1959: 46). We do not see the force of such counterexamples and we align ourselves with Bennett (1988: §5): a flash occurred because of how certain photons moved; a bang occurred because of how air ondulated; and so on.

${ }^{6}$ This is the view originated with Kim (1966) and Goldman (1970). Some authors might want to resist the identification and speak of supervenience instead: an event supervenes on the relevant property exemplification. We go along with the standard view, but what follows does not depend significantly on this decision.

${ }^{7}$ Ter Meulen (1987) objects to this suggestion on the following grounds. If we suppose, contrary to facts, that Brutus and Ceasar killed each other (simultaneously), then (A) would offer no explanation of the difference between the events described by 'Brutus killed Caesar' and 'Caesar killed Brutus'. We reply that if Brutus and Caesar are indeed the participants of these events, then (A) would imply their co-location while staying neutral (and correctly so) with respect to the issue of their distinctness. By contrast, if only part of Brutus was involved in the killing of Caesar or only part of Caesar involved in the killing of Brutus, then (A) (or rather ( $\mathrm{A}^{\prime}$ ) below) would correctly imply that the two events have distinct locations. 
${ }^{8}$ See e.g. Lombard (1986: 120f) (and the anticipation in Davidson 1967b: 124).

${ }^{9}$ This is the solution proposed by Lombard as well as others-e.g., Dretske (1967: 488) and Davidson (1969: 228).

${ }_{10}$ Philosophers who deny mereological extensionality (or the constitution-asidentity thesis) might want to say that the sinking had more than one participant, e.g., Titanic and the mereological sum of its parts. (See e.g. Carter 1989: 222f). But that would not have any bearing on the present issue, as the two (or more) entities in question would be perfectly co-located.

${ }^{11}$ Should the knife (say) be counted as a participant, too? We shall come back to this question in the final section. For the moment we assume that 'stabbed' is a binary predicate that fully connotes the relevant event-constitutive relation, hence that the event in question only involves two participants.

${ }^{12}$ For the sake of the example we shall suppose this to be a genuine possibility. As we mentioned, one could argue that a stabbing must be performed by a whole person, but this sort of suggestion can hardly be generalized to all cases (as illustrated by the Titanic example).

${ }^{13}$ Again, one could insist that (6') should be ruled out as inacceptable on the grounds that 'stabbed' imposes certain restrictions on the entities that can fill its argument places. (See previous note.) But then the solution would not be based exclusively on considerations of logical form but, rather, on a complicated interplay between logical form and lexical semantics.

${ }^{14}$ We have assumed that events are property exemplifications, following Kim (1966) and Goldman (1970). But we have not assumed the semantics that is usually associated with such metaphysics: the thesis that an event is an exemplification of a certain property, $P$, does not imply that any description of that event contains a predicate that fully connotes $P$. (So, again, we agree with Bennett 1988: Ch. 5).

${ }^{15}$ We are thankful to a referee for pointing this out.

${ }^{16}$ This would be in the spirit of Davidson's (1967a) treatment of adverbs.

${ }^{17}$ In the spirit of Rescher (1967).

${ }^{18}$ This paper grew out of portions of a paper presented by AV at the Conference on The Nature and Structure of States and Events (University of Southern California, Los Angeles, February 16, 2003). We are thankful to the discussants, Michael Glanzberg and Delia Graff, and to Jim Higgimbotham for their helpful comments on the conference paper, and to the referees of Philosophical Studies for their detailed remarks on the revised version.

\section{References}

Bennett, J., 1988, Events and Their Names, Oxford: Clarendon Press.

Brand, M., 1977, 'Identity Conditions for Events', American Philosophical Quarterly 14: 329-337.

Carter, W., 'Changing the Minimal Subject', Philosophical Studies 57: 217-226.

Davidson, D., 1967a, 'The Logical Form of Action Sentences', in N. Rescher (ed.), The Logic of Decision and Action, Pittsburgh: University of Pittsburgh Press, pp. 8195. 
Davidson, D., 1967b, 'Replies and Comments', in N. Rescher (ed.), The Logic of Decision and Action, Pittsburgh: University of Pittsburgh Press, pp. 123-129.

Davidson, D., 1969, 'The Individuation of Events', in N. Rescher (ed.), Essays in Honor of Carl G. Hempel, Dordrecht/Boston: Reidel, pp. 216-234.

Dretske, F., 1967, 'Can Events Move?', Mind 76: 479-492.

Fine, K., 1975, 'Vagueness, Truth and Logic', Synthese 30: 265-300.

Goldman, A., 1970, A Theory of Human Action, New York, Prentice-Hall.

Heller, M., 1990, The Ontology of Physical Objects: Four Dimensional Hunks of Matter, Cambridge: Cambridge University Press.

Kim, J., 1966, 'On the Psycho-Physical Identity Theory', American Philosophical Quarterly 3: 277-85.

Lewis, D. K., 1988, 'Vague Identity: Evans Misunderstood', Analysis 48: 128-130.

Lombard, L. B., 1986, Events. A Metaphysical Study, London: Routledge \& Kegan Paul.

Rescher, N., 1967, 'Aspects of Action', in N. Rescher (ed.), The Logic of Decision and Action, Pittsburgh: University of Pittsburgh Press, pp. 215-220.

Sider, T., 2001, Four-Dimensionalism. An Ontology of Persistence and Time, New York: Oxford University Press.

Sorensen, R. A., 1988, Blindspots, Oxford: Clarendon Press.

Strawson, P. F., 1959, Individuals: An Essay in Descriptive Metaphysics, London: Methuen.

Unger, P., 1979, 'There Are No Ordinary Things', Synthese 41: 117-154.

ter Meulen, A., 1987, 'Locating Events', in J. A. G. Groenendijk, D. de Jongh, and M. Stokhof (eds.), Foundations of Pragmatics and Lexical Semantics, Dordrecht: Foris Publications, pp. 27-40.

Varzi, A. C., 2001, 'Vagueness in Geography', Philosophy \& Geography 4: 49-65.

Varzi, A. C., 2002, 'Events, Truth, and Indeterminacy', The Dialogue 2: 241-264

Williamson, T., 1994, Vagueness, London: Routledge. 